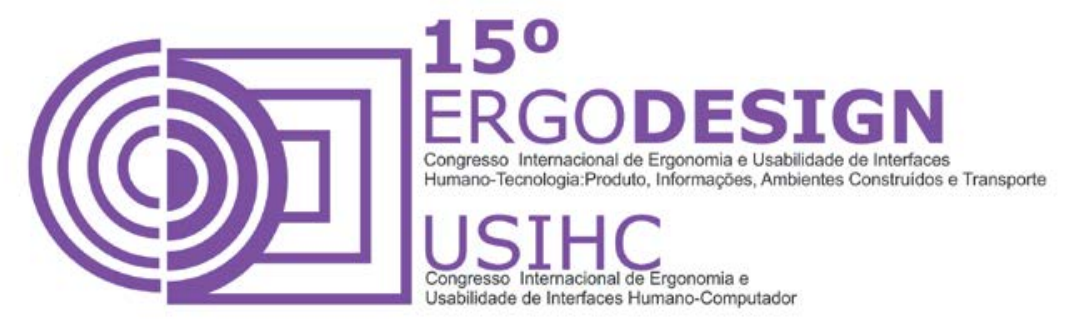

\title{
PROJETAÇÃO ERGONÔMICA DOS POSTOS DE TRABALHO DOS CARTEIROS DO CENTRO DE ENDEREÇAMENTO DE ENCOMENDAS SANTO CRISTO - RIO DE JANEIRO
}

\section{PROJECTING ERGONOMIC WORKING POSITIONS FOR POSTMEN OF THE "CENTRO DE ENDEREÇAMENTO DE ENCOMENDAS SANTO CRISTO - RIO DE JANEIRO"}

\author{
COSTA, Bianca Moura da (1); \\ COSTA, Cristina Leonise Chagas (2); \\ PINTO, Norma de Melo (3); \\ MENDONÇA, Paulo de Oliveira (4); \\ MORIM, Stela Curty Lima (5); \\ QUARESMA, Manuela (6);
}

(1) Pontifícia Universidade Católica do Rio de Janeiro, Especialização em Ergonomia e-mail:bianca.moura264@gmail.com

(2) Pontifícia Universidade Católica do Rio de Janeiro, Especialização em Ergonomia e-mail:cristina.leonise@gmail.com

(3) Pontifícia Universidade Católica do Rio de Janeiro, Especialização em Ergonomia e-mail:normadepintomelo@gmail.com

(4) Pontifícia Universidade Católica do Rio de Janeiro, Especialização em Ergonomia e-mail:professorpauloom@hotmail.com

(5) Pontifícia Universidade Católica do Rio de Janeiro, Especialização em Ergonomia e-mail:sclmorim@yahoo.com.br

(6) Pontifícia Universidade Católica do Rio de Janeiro, D. Sc. em Ergonomia e-mail:mquaresma@puc-rio.br 


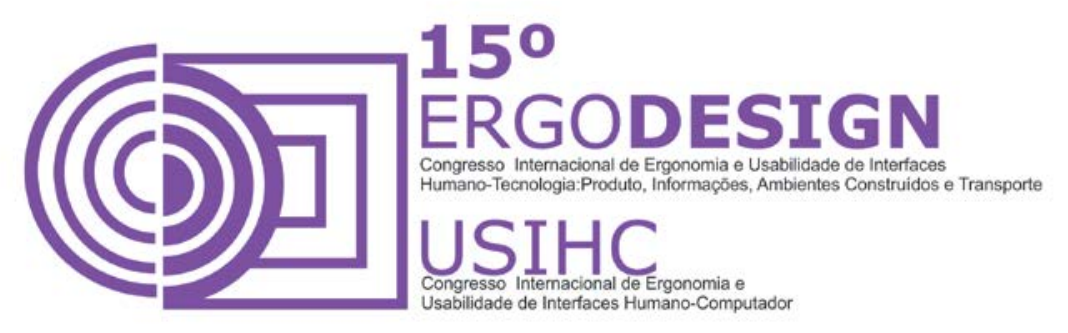

\title{
RESUMO
}

O objetivo dessa pesquisa foi elaborar um projeto e recomendações para um produto para setor da LOEC automática, no Centro de Endereçamento de Encomendas Santo Cristo RJ, diminuindo assim os efeitos prejudiciais à coluna vertebral. Ao longo desta pesquisa foram feitas análises da tarefa dos carteiros, as quais contribuíram para solução da projetação. Com a observação das adversidades foi testado um produto ergonômico de uso individual para a atividade dos carteiros.

Palavras-chave: Projetação, Ergonomia, Produto.

\begin{abstract}
The goal of this was to elaborate a project and recommendations for a product to the sector of LOEC in Central automatic Addressing Santo Cristo RJ, thus decreasing the harmful effects the spine. Throughout this research task analyses were done with postmen, which contributed to architectural solution. With The observation of hardships na ergonomic product was tested for single use for the activity of postmen.
\end{abstract}

Keywords: Design, Ergonomics, Product

\section{INTRODUÇÃO}

A projetação é parte do desenvolvimento de etapas da Análise Ergonômica (MORAES et al, 2009). O projeto desenvolvido através de desenhos antropométricos e testado no Centro de Endereçamento de Encomendas Santo Cristo (CEE Santo Cristo), localizado no Rio de Janeiro, Brasil. O CEE Santo Cristo possui 45 funcionários, sendo 40 carteiros, 1 carteiro motorizado, 1 gerente, 2 supervisores e 1 administrativo. O setor é responsável diariamente pela movimentação de entrega de encomendas e da entrega e coleta de malotes, e é subdividido em 29 distritos do Centro e Tijuca. Inicialmente as encomendas passam por um posto de trabalho denominado LOEC automática, onde são triados envelopes e caixas com até $30 \mathrm{Kg}$, que depois são designadas para seus respectivos distritos, manipulados por equipamento aramados com rodízios. Cada carteiro é responsável pela entrega de um distrito, dando prioridade ao Sedex 10 , que tem que ser entregue até às 10 horas do dia seguinte ao da postagem, com multa para a Empresa caso o horário não seja cumprido.

\section{OBJETIVO}

O objetivo deste projeto foi a criação de um mobiliário que pudesse minimizar os efeitos prejudiciais à musculatura, principalmente dos membros superiores e da coluna cervical permitindo obter redução do quadro álgico dos trabalhadores, de modo a poder aumentar a produtividade, no setor da LOEC automática.

\section{MATERIAIS E MÉTODOS}

Os métodos aplicados foram observações sistemáticas, através de registros de frequência de acionamentos, tomadas de informação e questionários com o intuito de obter relatos das 


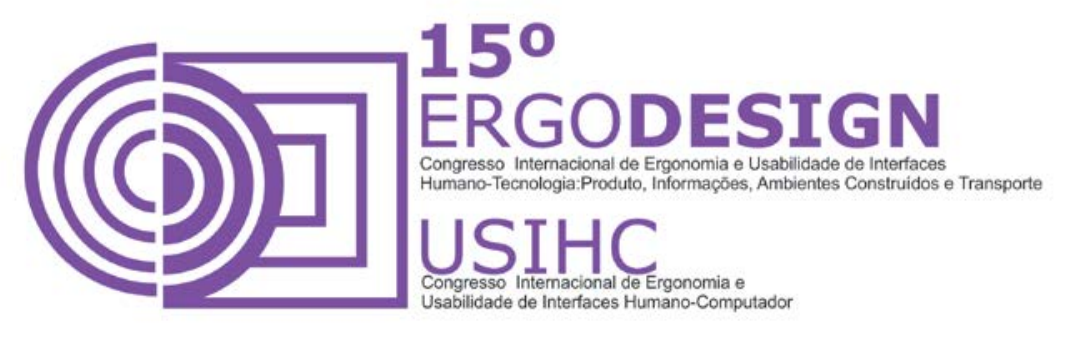

necessidades dos carteiros. De acordo com a metodologia Moraes e Mont'Alvão (2009), a análise de posturas em uma avaliação de comportamento pode contribuir para várias necessidades dos usuários. A partir de resultados foi feita a recomendação de um suporte de monitor, com a substituição do monitor simples por um monitor touch screen de 15'.

\section{REFERÊNCIAL TEÓRICO}

Segundo Filho e Lopes (2001), a qualidade de vida do trabalho de uma pessoa é influenciada por vários fatores como físico, emocional, social, intelectual, espiritual e a ocupacional. Estes fatores, são elementos que ajudam de acordo com a circunstância em que o trabalho do indivíduo é executado. Hoje em dia no trabalho, tem-se o somatório das altas cargas de trabalho que se inserem consequentemente prejudicando os serviços prestados e a saúde de um indivíduo.

A Ergonomia é o estudo dos seres humanos no seu ambiente de trabalho (MORAES et al, 2009). A implantação da Ergonomia contribui para melhorar o dia a dia dos trabalhadores, tornando móveis, meios de transportes, ambientes construídos e tecnológicos agradáveis, eficientes e seguros sua utilização (IIDA, 2005).

Hoje em dia os altos índices de informações prejudicam funcionários e altos gastos nas empresas, com erros humanos (IIDA,2005). Isso tudo pode causar lesões, doenças ou até mesmo a morte.

As medidas antropométricas influenciam nas indústrias e ajudam adaptar os produtos nas dimensões corporais da população usuária (BRIGDER, 1995 apud QUARESMA, 2001, p.8). Baseado nos estudos ergonômicos foi utilizado a publicação do levantamento antropométrico americano do Diffrient, 1981, que abrange 95\% da população.

\section{RESULTADOS}

Analisando os registros das frequências das atividades de acionamento (ANEXO 1), tomada de informações (ANEXO 2) e observações de fotografias (ANEXO 3) registrados no local do CEE Santo Cristo, observou-se a repetitividade da flexo-extensão e rotação da coluna vertebral na LOEC automática. Essas atividades podem gerar dores ou cansaço muscular nos carteiros. Como solução o produto proposto para a área de acionamento foi a substituição do monitor, teclado, mouse pelo monitor touch screen de 15" de modo a evitar a repetitividade da flexoextensão e rotação da coluna vertebral. Além disso, foi projetado - um suporte para o monitor (ANEXO 5) utilizando os percentis antropométricos extremos (mulher 2,50 e homem 97,50) (ANEXO 4), de forma a adaptar o acionamento e visualização do monitor por ambos usuários.

\section{CONCLUSÃO}

A pesquisa foi realizada para testar no posto da LOEC automática com o fim de permitir o 


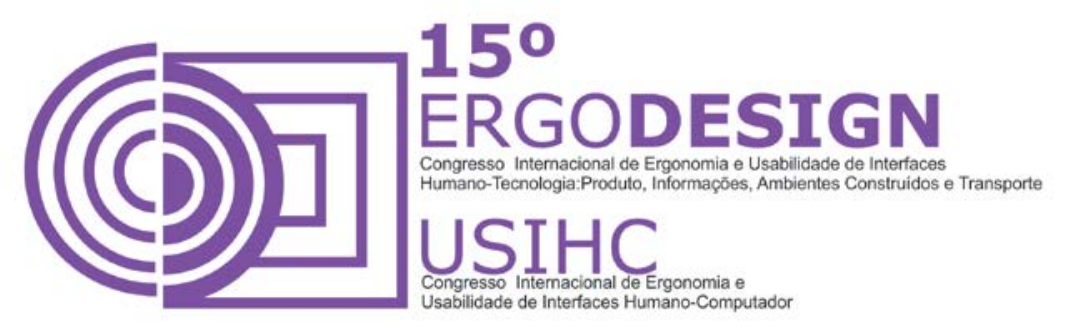

desenvolvimento de um trabalho mais ergonômico. Sugerindo produtos que já existem no mercado, solucionando ou minimizando os problemas com os modelos de teste no do CCESanto Cristo para serem implantados.

\section{REFERENCIAIS BIBLIOGRÁFICAS}

FILHO, Gilséen Ivan Regis; LOPES, Cristina Mônica - Qualidade de vida no trabalho: a empresa holística e a ecologia empresarial. São Paulo, 2001. Disponível em http://www.rausp.usp.br/busca/artigo.asp?num artigo=276 . Acessado em Janeiro de 2015.

IIDA, I. Ergonomia - Projeto e Produção. São Paulo: Edgar Blucher, 2005.

MORAES, Anamaria de. MONT'ALVÃO, Claudia. Ergonomia: conceitos e aplicações. Rio de Janeiro: 2AB, 2009 (4 edição, ampliada).

QUARESMA, M. Manuela. A Aplicação de dados antropométricos em projetos de design: como projetar corretamente produtos ergonômicos. Rio de Janeiro: PUC - RJ, 2001 (Dissertação, Mestrado em Design).

\section{ANEXO 1}

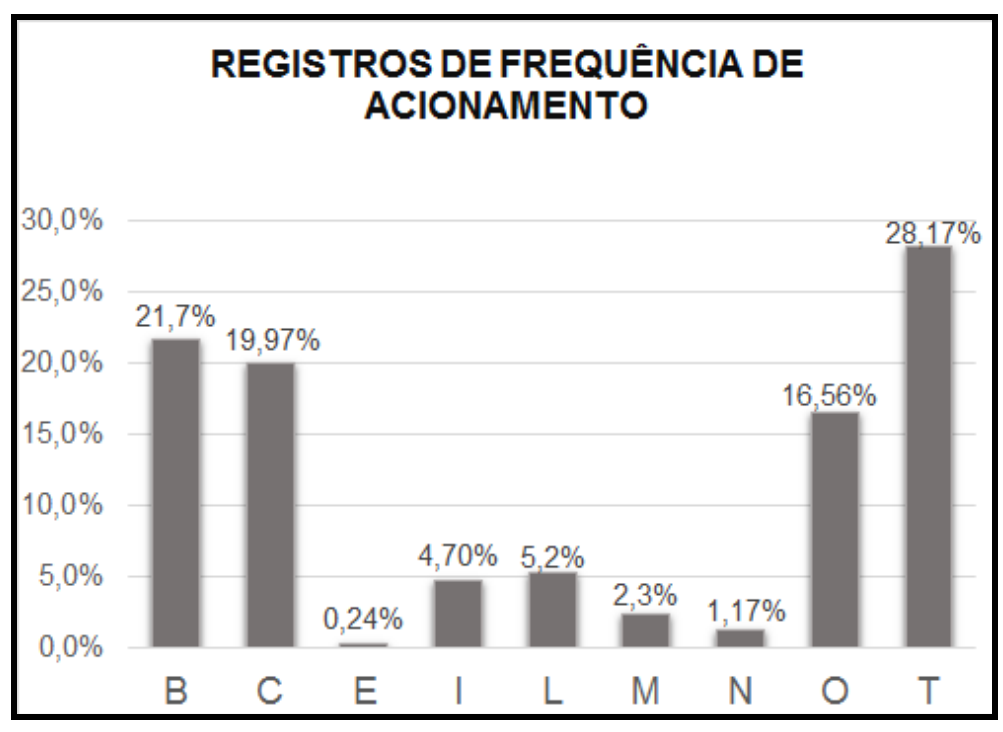

Figura 1 - Gráfico dos registros frequência de acionamentos dos usuários no setor da LOEC automática. Fonte: Autores 


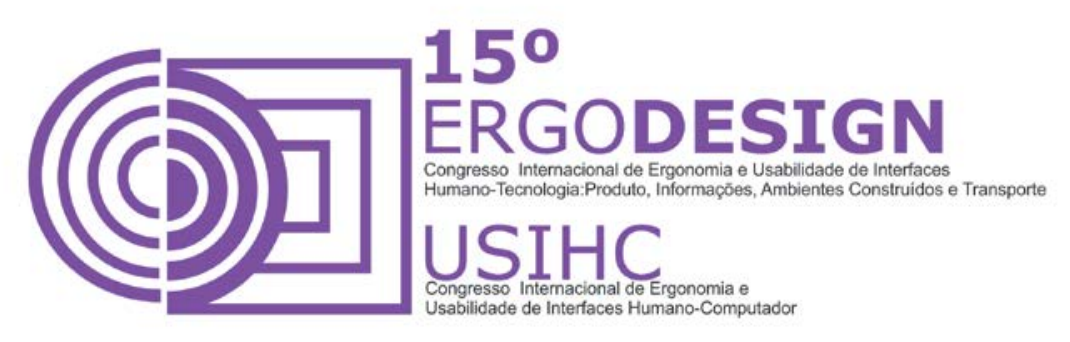

\begin{tabular}{|c|c|}
\hline \multicolumn{2}{|c|}{ LEGENDA } \\
\hline B & PEGAR O LEITOR DE CÓD. DE BARRAS \\
\hline C & PEGAR A ENCOMENDA \\
\hline E & ESCREVER \\
\hline I & PEGAR A ETIQUETA NA IMPRESSORA \\
\hline L & COLAR A ETIQUETA \\
\hline M & PEGAR NO MOUSE \\
\hline N & PEGAR A CANETA \\
\hline O & OUTROS \\
\hline T & DIGITAR NO TECLADO \\
\hline
\end{tabular}

Figura 2 - Legenda dos símbolos do gráfico acima

ANEXO 2

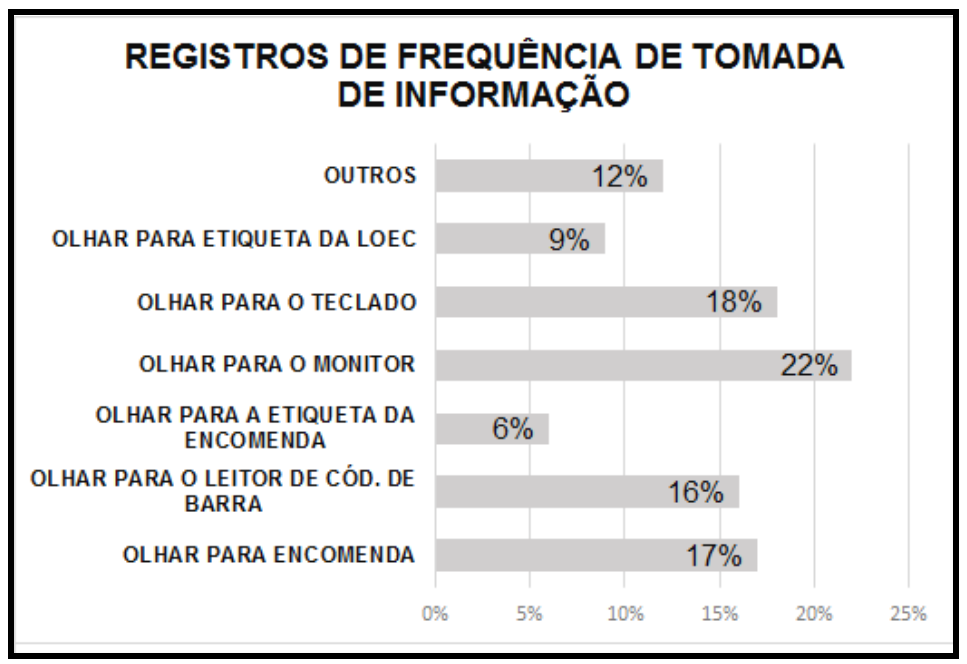

Figura 3 - Gráfico dos registros frequência dos campos visuais dos usuários no setor da LOEC automática. Fonte: Autores

ANEXO 3 

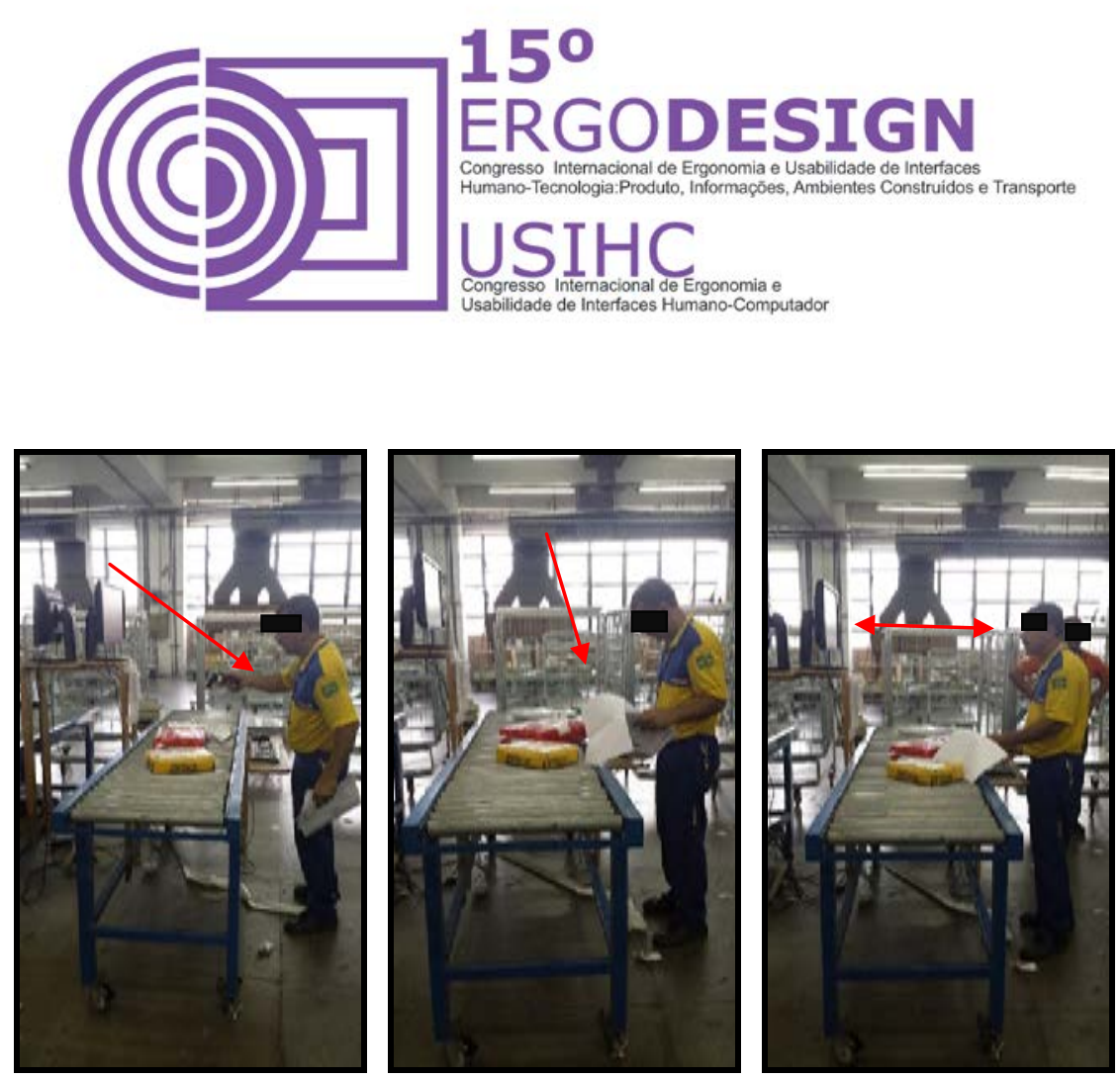

Figura 3 - Algumas tarefas dos usuários na LOEC automática Fonte: Autores
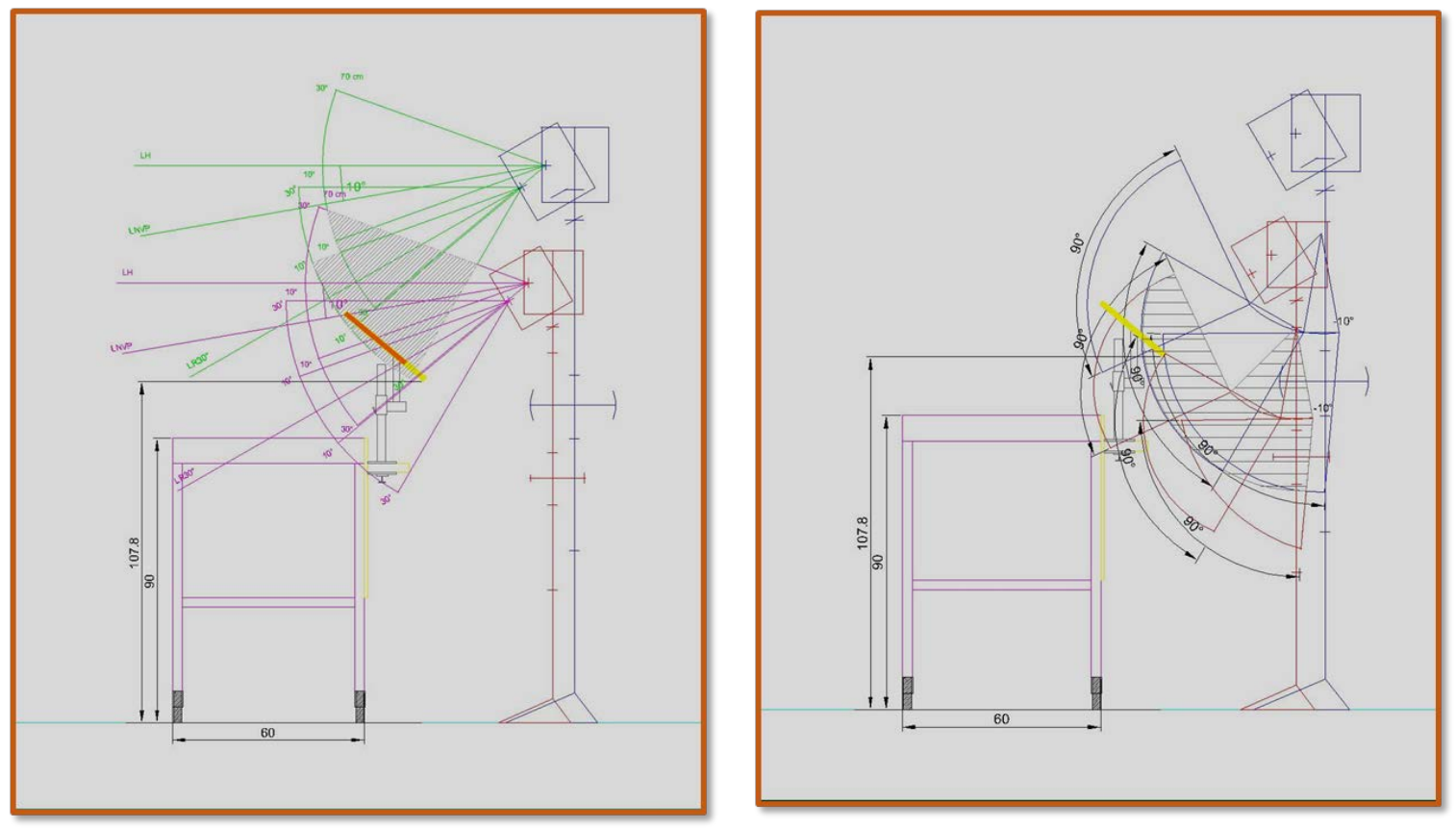

Figura 5 - Dimensões dos campos visuais e acionais manequins antropométricos Fonte: Autores 


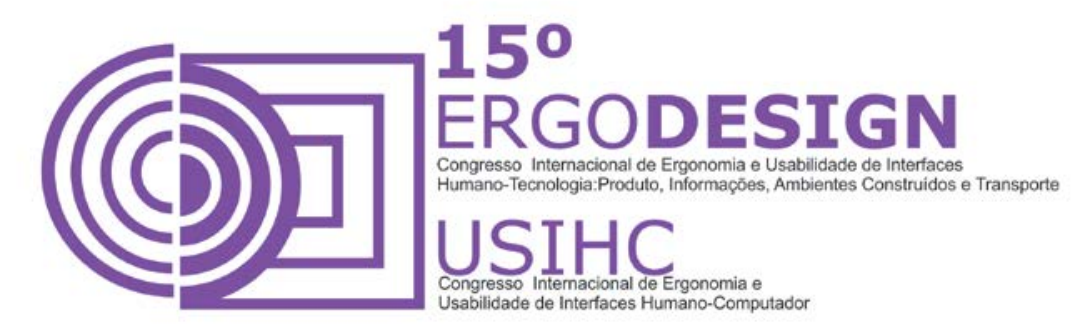

ANEXO 5

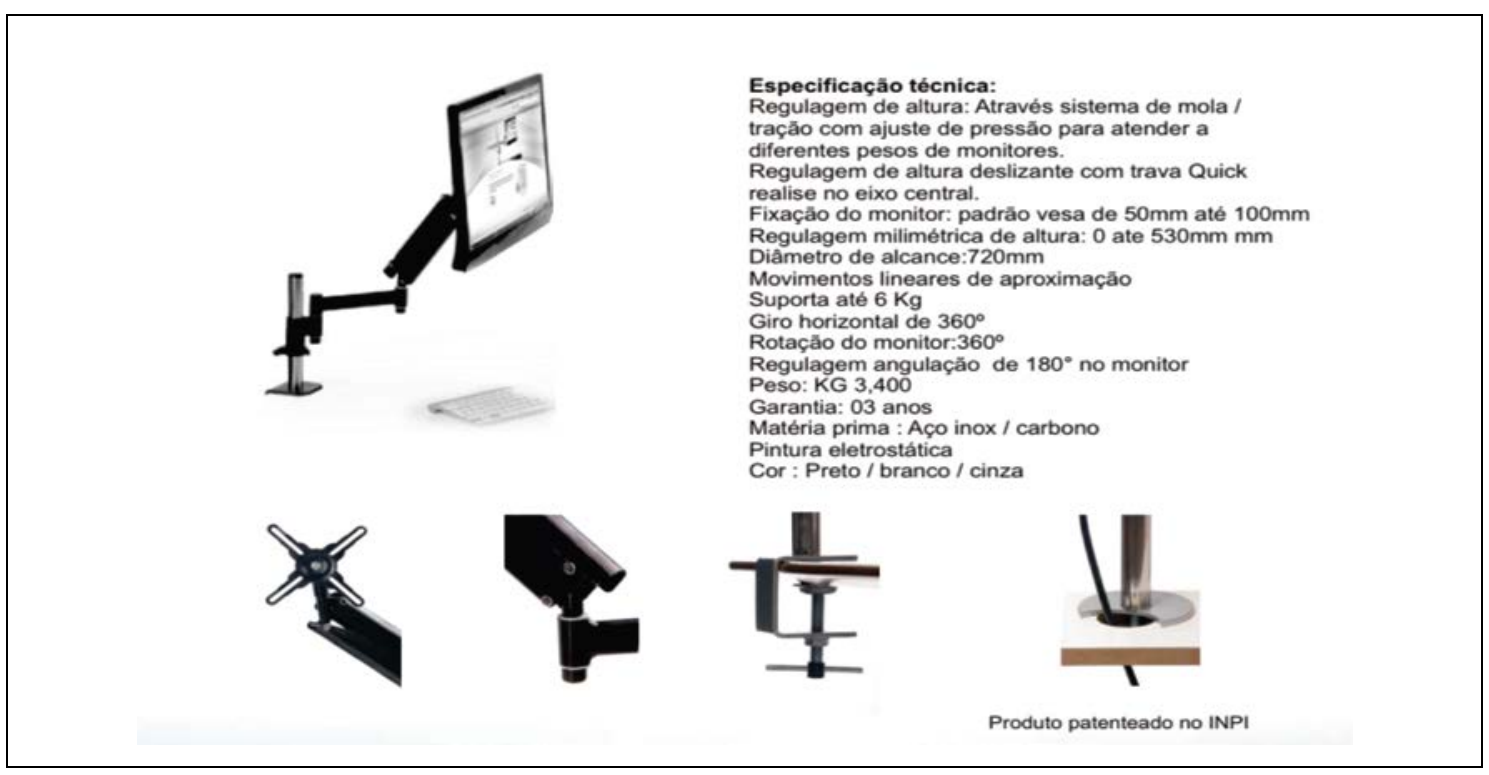

Figura 6: Suporte para monitor

Fonte: Ergoline. Foto concedida pelo fabricante 